\title{
Proton motive force-dependent efflux of tetracycline in clinical isolates of Helicobacter pylori
}

\begin{abstract}
Correspondence
Tahereh Falsafi

tfalsafi@yahoo.com
\end{abstract}

Received 6 March 2009

Accepted 25 June 2009

\author{
Mona Anoushiravani, ${ }^{1}$ Tahereh Falsafi ${ }^{1}$ and Vahid Niknam ${ }^{2}$ \\ ${ }^{1}$ Department of Biology, Alzahra University, Tehran, Iran \\ ${ }^{2}$ Department of Biology, Tehran University, Tehran, Iran
}

\begin{abstract}
The aim of this study was to evaluate the role of proton motive force (PMF)-dependent efflux in resistance of Helicobacter pylori to tetracycline (Tet). Tet MIC was determined by agar dilution in the presence and absence of carbonyl cyanide $m$-chlorophenylhydrazone (CCCP), an inhibitor of PMF. Antibiotic accumulation was conducted in the presence or absence of CCCP and the fluorescence of the accumulated antibiotic was measured by spectrofluorometry. In the presence of CCCP, antibiotic accumulation was increased by $2-17$-fold in $17 / 20$ Tet $^{r}$ isolates and by 310-fold in four of five high-level-resistant mutants. Correlation was observed between this increase and diminution of MIC with CCCP. PMF-dependent efflux mechanisms therefore appear to play an important role in the resistance of clinical isolates of $H$. pylori to Tet.
\end{abstract}

\section{INTRODUCTION}

The antibiotics commonly used to eradicate Helicobacter pylori infection include amoxicillin (Amx), clarithromycin (Cla), tetracycline (Tet) and metronidazole (Mtz), plus a proton pump inhibitor (Graham \& Qureshi, 2000; Loffeld \& Fijen, 2003). As Tet can cause staining of the permanent teeth and interfere with bone growth, it is not recommended for young children. However, $\mathrm{Tet}^{\mathrm{R}} H$. pylori strains have increasingly been isolated in both adults and children, especially in developing and Asian countries (Kwon et al., 2000; Mendonca et al., 2000; Wu et al., 2000, 2005).

Tet is an inhibitor of protein synthesis with a broad spectrum of activity against both Gram-negative and Gram-positive bacteria. It binds to the $30 \mathrm{~S}$ ribosomal subunit and blocks the binding of aminoacyl-tRNA, preventing the synthesis of nascent peptide chains (Chopra \& Roberts, 2001). The mechanisms of bacterial resistance to Tet have been widely studied in many bacteria. Four different Tet resistance mechanisms have been described: (i) a defect in the uptake of the antibiotic; (ii) an increase in its efflux; (iii) decreased antibiotic binding, either by changes in ribosomal protection proteins or by mutations in the 16S rRNA tetracycline-binding site; and (iv) enzymic inactivation of Tet (Chopra \& Roberts, 2001; Nikaido, 1998). In H. pylori, involvement of the first three mechanisms in resistance to Tet has been proposed; however, the best-studied mechanism remains mutation in the 16S rRNA gene, which is based on a single, double or triple base-pair substitution in adjacent $16 \mathrm{~S}$ rRNA gene

Abbreviations: Amx, amoxicillin; CCCP, carbonyl cyanide $m$-chlorophenylhydrazone; Cla, clarithromycin; Mtz, metronidazole; PMF, proton motive force; Tet, tetracycline. residues (Dailidiene et al., 2002; Glocker et al., 2005; Kwon et al., 2000; Lawson et al., 2005; Trieber \& Taylor, 2002). These mutations are located in the primary binding site of tetracycline, and probably affect the affinity of the drug for the ribosome, thus reducing its inhibitory effect (Lawson et al., 2005; Nonaka et al., 2005).

In many Gram-negative bacteria, resistance to tetracycline is also related to an energy-dependent efflux of Tet across the cell membrane (Chopra \& Roberts, 2001; Nikaido, 1998). Although several investigators have studied the probable role of active efflux in the intrinsic resistance of H. pylori to antibiotics (Bina et al., 2000; Kutschke \& de Jonge, 2005; Li \& Dannelly, 2006; van Amsterdam et al., 2005), there are no data concerning the precise role of this mechanism in resistance to Tet. In a recent study, $\mathrm{Wu}$ et al. (2005) examined whether Tet resistance was associated with an energy-coupled active efflux mechanism. In this work, they were not able to observe the role of active efflux in resistance to Tet, but they proposed alteration of membrane permeability as a possible mechanism responsible for resistance of $H$. pylori to Tet.

The aim of our study was to evaluate the role of proton motive force (PMF)-dependent efflux in the primary resistance of $H$. pylori to Tet in clinical isolates obtained from children.

\section{METHODS}

Bacterial strains and culture conditions. $H$. pylori isolates were obtained by screening of $112 \mathrm{H}$. pylori strains isolated during 19972008 from 250 children at the Children Medical Center of Tehran. Bacteria were routinely grown on modified Campy blood agar and Belo Horizonte agar (Merck) as described previously (Falsafi et al., 
2007). Colonies were identified by Gram staining, biochemical tests and PCR using H. pylori-specific primers 16sRNA (Falsafi et al., 2009) and ureC (Cinnagen), as described previously (Falsafi et al., 2009).

MIC determination. Susceptibility to Tet (Sigma) was determined by establishing the MIC value according to the recommendations of the Clinical and Laboratory Standards Institute (Best et al., 2003). Plates were prepared with Mueller-Hinton (MH) agar (Merck) plus $7 \%$ fresh sheep blood and Tet at a concentration of $0.5-128 \mathrm{mg}^{-1} . H$. pylori isolates were grown for 3 days on modified Campy blood agar plates, harvested and suspended in sterile saline to obtain a McFarland 2 standard $\left(\sim 1 \times 10^{8}-2 \times 10^{8}\right.$ c.f.u. $\left.\mathrm{ml}^{-1}\right)$. The $\mathrm{MH}$ agar plates were spread with bacterial suspension and incubated for $2-$ 3 days at $37{ }^{\circ} \mathrm{C}$ under microaerobic conditions. Antibiotic-free plates were inoculated during each series of tests to confirm the validity of the inoculum and to observe the presence of any contamination. $H$. pylori ATCC 26695 was used as the quality-control organism and measurements were repeated three times to confirm the results. The resistance cut-off point for Tet was an MIC of $\geqslant 4 \mathrm{mg} \mathrm{l}^{-1}$ (King, 2001; Lawson et al., 2005).

Determination of MIC was also performed in the presence of carbonyl cyanide $m$-chlorophenylhydrazone (CCCP; Sigma), an inhibitor that disrupts the proton gradient across the membrane. For this purpose, inoculated plates were dried for $5 \mathrm{~min}$ and discs containing $30 \mu \mathrm{l} \mathrm{CCCP}$ at a concentration of $100 \mu \mathrm{M}$ were placed on the agar surface. A control plate containing $100 \mu \mathrm{M}$ CCCP showed that this concentration of CCCP did not produce any collapse of bacterial growth. The plates were incubated for 2 days, after which the MIC was defined as the lowest concentration of antibiotic that showed growth inhibition around the CCCP disc.

Determination of cross-resistance to Mtz, Amx and Cla by the disc diffusion method. All $\mathrm{Tet}^{\mathrm{R}} \mathrm{H}$. pylori isolates were also tested by the disc diffusion method for susceptibility to Tet, Mtz, Cla and Amx. Discs containing Tet $(30 \mu \mathrm{g})$, Amx $(30 \mu \mathrm{g})$, Mtz $(5 \mu \mathrm{g})$ and Cla $(15 \mu \mathrm{g})$ were purchased from Himedia. Five microlitres of bacterial suspension at a concentration of a McFarland 2 standard was spread on sheep blood $\mathrm{MH}$ agar plates, the discs were added and the diameters of the zones of inhibition were measured after 3 days. Quality control was ensured by using the following organisms: Pseudomonas aeruginosa ATCC 27853, Staphylococcus aureus ATCC 29213, Escherichia coli ATCC 25922 and H. pylori ATCC 26695.

Inhibition zones of $\geqslant 30, \geqslant 14, \geqslant 10$ and $\geqslant 14 \mathrm{~mm}$ were interpreted as $\mathrm{H}$. pylori resistance to Tet, Mtz, Cla and Amx, respectively (Lawson et al., 2005; McNulty et al., 2002).

Accumulation assay. Accumulation of Tet in $H$. pylori was determined as described previously (Lin et al., 2002) with some modifications. H. pylori strains were grown on Campy blood or $\mathrm{MH}$ agar plates for 2 days, harvested and resuspended in $50 \mathrm{mM}$ sodium phosphate buffer ( $\mathrm{pH} 7.2$ ) with a density equivalent to a McFarland 4 standard $\left(\mathrm{OD}_{600}=0.6\right)$ corresponding to $1.2 \times 10^{9}$ c.f.u. $\mathrm{ml}^{-1}$. The cell suspension was incubated for $10 \mathrm{~min}$ at $37{ }^{\circ} \mathrm{C}$ and antibiotic-uptake assays were initiated by the addition of Tet to $500 \mu \mathrm{l}$ bacterial cell suspension at a final concentration of $20 \mu \mathrm{g} \mathrm{ml}^{-1}$. At $10 \mathrm{~min}$ after antibiotic addition, CCCP was added to half of the reaction mixture at a final concentration of $200 \mu \mathrm{M}$ and the other half was used as a control (no CCCP). After $20 \mathrm{~min}$, each of the collected samples was diluted in $1.5 \mathrm{ml}$ ice-cold sodium phosphate buffer and centrifuged for $15 \mathrm{~min}$ at $6000 \mathrm{~g}$ at $4{ }^{\circ} \mathrm{C}$. The supernatants were discarded and the pellets were resuspended in $2 \mathrm{ml} 0.1 \mathrm{M}$ glycine hydrochloride $(\mathrm{pH} 3.0)$ and shaken at $25{ }^{\circ} \mathrm{C}$ for $16 \mathrm{~h}$ to extract the accumulated antibiotic. After centrifugation at $6000 \mathrm{~g}$ for $15 \mathrm{~min}$, the supernatant was used to measure the fluorescence of Tet with a Shimadzu RF 5000 spectrofluorometer (Shimadzu Scientific Instruments) at excitation and emission wavelengths of 400 and $450 \mathrm{~nm}$, respectively. The concentration of Tet in the supernatant was measured by comparison with a standard curve of Tet in $0.1 \mathrm{M}$ glycine hydrochloride. The results were expressed as ng Tet (mg dry weight bacteria $)^{-1}$.

Selection of $\boldsymbol{H}$. pylori high-level-resistant mutants. Five clinical $H$. pylori isolates that were weakly or moderately resistant to Tet were grown on Campy blood agar plates and consecutively subcultured on plates supplemented with increasing concentrations of Tet. After growth, one colony of each isolate that was capable of growth at the highest concentration of Tet was picked and tested for a stable high MIC. These selected mutants were subjected to the studies described above for clinical isolates.

\section{RESULTS AND DISCUSSION}

A total of $112 \mathrm{H}$. pylori isolates were screened and $20 \mathrm{Tet}^{\mathrm{R}}$ isolates with MICs of $4-64 \mathrm{mg} \mathrm{l}^{-1}$ were isolated. Two isolates susceptible to Tet, Mtz, Amx and Cla were used as negative controls $(1 \mathrm{~B}$ and $17 \mathrm{~B})$. Tet $^{\mathrm{R}}$ isolates were classified as low-, intermediate- or high-level Tet ${ }^{\mathrm{R}}$ according to their MICs. Among them, 14 (70\%) showed cross-resistance to Mtz, but no resistance was observed for Amx and Cla (Table 1). In the presence of CCCP, the Tet MIC was decreased by one to two dilutions for 17/20 isolates (Table 2). These $17 \mathrm{Tet}^{\mathrm{R}}$ isolates with a CCCP effect on MIC showed PMF-dependent efflux of Tet manifested by a

Table 1. Tet MICs in relation to cross-resistance to Amx, Mtz and Cla

Resistance to Tet was determined by the agar dilution and disc diffusion methods. S, Sensitive; R, resistant; I, intermediate.

\begin{tabular}{|c|c|c|c|}
\hline Strain no. & $\operatorname{Amx}$ & Mtz & Cla \\
\hline \multicolumn{4}{|c|}{ Low-level resistance (MIC $4-8 \mu \mathrm{g} \mathrm{ml}^{-1}$ ) } \\
\hline $12 \mathrm{M}$ & S & S & S \\
\hline $15 \mathrm{M}$ & $S$ & I & S \\
\hline $27 \mathrm{M}$ & S & S & S \\
\hline $10 \mathrm{II}$ & $S$ & I & $S$ \\
\hline $18 \mathrm{~B}$ & $S$ & $\mathrm{R}$ & $S$ \\
\hline $58 \mathrm{II}$ & $S$ & $\mathrm{R}$ & $S$ \\
\hline $23 \mathrm{M}$ & S & S & S \\
\hline \multicolumn{4}{|c|}{ Moderate resistance $\left(\right.$ MIC $16 \mu \mathrm{g} \mathrm{ml}^{-1}$ ) } \\
\hline $19 \mathrm{M}$ & S & S & S \\
\hline $20 \mathrm{M}$ & $S$ & $\mathrm{R}$ & I \\
\hline $24 \mathrm{M}$ & S & $\mathrm{R}$ & $S$ \\
\hline $25 \mathrm{M}$ & S & $\mathrm{R}$ & I \\
\hline $26 \mathrm{M}$ & $S$ & $\mathrm{R}$ & $S$ \\
\hline $28 \mathrm{M}$ & $S$ & $\mathrm{R}$ & $S$ \\
\hline $21 \mathrm{D}$ & $S$ & $\mathrm{R}$ & S \\
\hline $2 \mathrm{~N}$ & S & $\mathrm{R}$ & I \\
\hline \multicolumn{4}{|c|}{$\begin{array}{l}\text { High-level resistance (MIC 32-64 } \mu \mathrm{g} \\
\mathrm{ml}^{-1} \text { ) }\end{array}$} \\
\hline $17 \mathrm{M}$ & $S$ & $\mathrm{R}$ & $S$ \\
\hline $18 \mathrm{M}$ & $S$ & $\mathrm{R}$ & $S$ \\
\hline $21 \mathrm{M}$ & I & $\mathrm{R}$ & $S$ \\
\hline $1 \mathrm{~N}$ & $S$ & $\mathrm{R}$ & I \\
\hline $3 \mathrm{~N}$ & I & $\mathrm{R}$ & $S$ \\
\hline
\end{tabular}


Table 2. Tet MICs in relation to accumulation results in the presence and absence of CCCP

\begin{tabular}{|c|c|c|c|c|c|}
\hline \multirow[t]{2}{*}{ Strain no. } & \multicolumn{2}{|c|}{ MIC } & \multicolumn{2}{|c|}{ Accumulation ${ }^{*}$} & \multirow[t]{2}{*}{ Increase $\dagger$} \\
\hline & $-\mathrm{CCCP}$ & $+\mathrm{CCCP}$ & $-\mathrm{CCCP}$ & $+\mathrm{CCCP}$ & \\
\hline $12 \mathrm{M}$ & 8 & 4 & 0.08 & 1.33 & 16.6-fold \\
\hline $15 \mathrm{M}$ & 8 & 4 & 0.69 & 1.14 & 1.5 -fold \\
\hline $27 \mathrm{M}$ & 8 & 4 & 0.90 & 2.70 & 3-fold \\
\hline $58 \mathrm{II}$ & 4 & 4 & 0.48 & 0.52 & No change \\
\hline $23 \mathrm{M}$ & 4 & 2 & 0.38 & 0.83 & 2-fold \\
\hline \multicolumn{6}{|c|}{ Moderate resistance } \\
\hline $19 \mathrm{M}$ & 16 & 8 & 1.46 & 3.45 & 2.4-fold \\
\hline $20 \mathrm{M}$ & 16 & 8 & 0.14 & 0.29 & 2-fold \\
\hline $21 \mathrm{D}$ & 16 & 8 & 0.22 & 0.45 & 2-fold \\
\hline $2 \mathrm{~N}$ & 16 & 16 & 1.64 & 1.84 & No change \\
\hline \multicolumn{6}{|c|}{ High-level resistance } \\
\hline $17 \mathrm{M}$ & 32 & 8 & 0.33 & 2.27 & 7-fold \\
\hline $18 \mathrm{M}$ & 32 & 8 & 0.32 & 0.67 & 2-fold \\
\hline $21 \mathrm{M}$ & 32 & 8 & 0.14 & 0.27 & 2-fold \\
\hline $1 \mathrm{~N}$ & 32 & 8 & 0.39 & 2.5 & 6-fold \\
\hline $3 \mathrm{~N}$ & 64 & 16 & 0.65 & 2.34 & $\sim 4$-fold \\
\hline \multicolumn{6}{|l|}{ Susceptible } \\
\hline $1 \mathrm{~B}$ & 0.5 & ND & 0.05 & 0.055 & No change \\
\hline $17 \mathrm{~B}$ & 1 & ND & 0.10 & 0.11 & No change \\
\hline
\end{tabular}

ND, Not determined.

${ }^{\star}$ Measured as ng Tet (mg dry weight bacteria) ${ }^{-1}$.

$\dagger$ Fold increase in concentration of accumulated Tet in the presence of CCCP.

2-17-fold increase in the amount of Tet (mg dry weight bacteria) ${ }^{-1}$ in the presence of CCCP (Table 2). No significant difference was observed between low-, intermediate- and high-level Tet $^{\mathrm{R}}$ isolates regarding the amount of energy-dependent efflux of Tet. High-level Tet ${ }^{\mathrm{R}}$ mutants that were selected on the basis of growth on increasing concentrations of Tet and that had stable MICs showed an MIC that was increased by one to three dilutions. Their cross-resistance profiles remained unchanged, except for mutant $23 \mathrm{M}$, which acquired resistance to $\mathrm{Mtz}$ during passage (Table 3). In the presence of CCCP, the MIC for Tet was decreased by two dilutions for all of the high-level Tet $^{\mathrm{R}}$ mutants, except for the $23 \mathrm{M}$ mutant, which remained unchanged. In the presence of CCCP, the concentration of accumulated Tet in the mutants increased 3-10-fold compared with their parental strains, except for $23 \mathrm{M}$, which showed a decrease (Table 3).

Although Tet resistance is rarer in H. pylori than in other bacteria, its emergence varies according to geographical area. For example, in Western countries, Tet resistance is rare (Osato et al., 2001), but in Japan and Korea, 5-7\% resistance has been observed, and in China high levels of resistance ( $59 \%$ ) have been found (Wu et al., 2000). We screened 112 isolates and found $18 \%$ resistance to Tet with MICs ranging from 4 to $64 \mathrm{mg} \mathrm{l}^{-1}$. Seventy per cent of these isolates were cross-resistant to $\mathrm{Mtz}$, similar to results observed in Korea (Kim et al., 2001). Although Trieber \& Taylor (2002) have proposed that the known Tet $^{\mathrm{R}}$ loci are not genetically linked to the $\mathrm{Mtz}^{\mathrm{R}}$ locus, it is difficult to extrapolate this suggestion to all cases, as one mutant $(23 \mathrm{M}$ mutant) that was co-resistant to both antibiotics was selected in this work.

In the present work, a significant increase in the concentration of accumulated Tet in the presence of CCCP was observed for $17 \mathrm{Tet}^{\mathrm{R}}$ isolates, suggesting that efflux pumps energized by PMF were functional in these isolates. However, no significant difference was observed between low-, intermediate- and high-level $\mathrm{Tet}^{\mathrm{R}}$ isolates regarding the amount of active efflux (Table 2). This suggests that the activity of efflux pumps may not be 
Table 3. Tet MICs, cross-resistance and accumulation of five high-level Tet ${ }^{\mathrm{R}}$ mutants compared with the parental isolates

\begin{tabular}{|c|c|c|c|c|c|c|}
\hline \multirow[t]{2}{*}{ Strain no. } & \multicolumn{2}{|c|}{ Tet MIC } & \multirow[t]{2}{*}{ Cross-resistance to Amx-Mtz-Cla } & \multicolumn{2}{|c|}{ Accumulation ${ }^{\star}$} & \multirow[t]{2}{*}{ Increase $\dagger$} \\
\hline & $-\mathrm{CCCP}$ & $+\mathrm{CCCP}$ & & $-\mathrm{CCCP}$ & $+\mathrm{CCCP}$ & \\
\hline $18 \mathrm{M}$ & 32 & 8 & S R S & 0.32 & 0.67 & 2-fold \\
\hline $18 \mathrm{M}-\mathrm{mut}$ & 64 & 16 & S R S & 0.37 & 1.97 & 5.3-fold \\
\hline $20 \mathrm{M}$ & 16 & 32 & S R I & 0.14 & 0.29 & 2-fold \\
\hline 20M-mut & 64 & 32 & S R I & 0.29 & 1.5 & 5 -fold \\
\hline $21 \mathrm{M}$ & 32 & 8 & I R S & 0.14 & 0.27 & 2-fold \\
\hline 21M-mut & 64 & 16 & S R I & 0.75 & 2.6 & 3.5-fold \\
\hline $23 \mathrm{M}$ & 4 & 2 & S S S & 0.38 & 0.83 & 2-fold \\
\hline 23M-mut & 32 & 32 & S R S & 0.16 & 0.32 & 2-fold $\ddagger$ \\
\hline $25 \mathrm{M}$ & 16 & 8 & S R I & 0.26 & 0.55 & 2-fold \\
\hline 25M-mut & 32 & 8 & S R S & 0.35 & 2.4 & 7-fold \\
\hline
\end{tabular}

${ }^{\star}$ Measured as ng Tet (mg dry weight bacteria $)^{-1}$.

$\dagger$ Fold increase in concentration of accumulated Tet in the presence of CCCP.

$\ddagger$ A decrease of $\sim 2.5$-fold (in the presence of CCCP) compared with the $23 \mathrm{M}$ parent strain.

related to the MIC and that more than one mechanism may be responsible for the development of high-level Tet resistance. The increase in amount of proton-dependent efflux in four of the five high-level-resistant mutants (Table 3) may indicate that, during passage, the effect of pressure of the antibiotic selected the strains with the more active efflux pumps. However, during step-wise growth on Tet, the MIC of strain $23 \mathrm{M}$ was increased from 4 to 32 , although its efflux activity was widely decreased (Table 3 ), which may relate to alteration of its permeability. The phenotype observed in the case of this mutant is similar to those observed by $\mathrm{Wu}$ et al. (2005), suggesting that decreased accumulation of Tet may be one of the Tet resistance mechanisms. However, the key finding of this work is that CCCP, an inhibitor of PMF, increased Tet accumulation in clinical isolates and in high-level-resistant mutants. This is different from the findings of $\mathrm{Wu}$ et al. (2005): whilst they showed decreased accumulation in resistant strains, they were not able to demonstrate the PMF-dependent efflux of Tet.

Thus, the results of the present study provide data in favour of active efflux as a significant mechanism in the resistance of clinical isolates of $H$. pylori to Tet.

\section{ACKNOWLEDGEMENTS}

This work was supported by Alzahra University, Tehran, Iran.

\section{REFERENCES}

Best, L. M., Haldane, D. J. M., Keelan, M., Taylor, D. E., Thomson, A. B. R., Loo, V., Fallone, C. A., Lyn, P., Smaill, F. M. \& other authors (2003). Multilaboratory comparison of proficiencies in susceptibility testing of Helicobacter pylori and correlation between agar dilution and E test methods. Antimicrob Agents Chemother 47, 31383144.
Bina, J. E., Alm, R. A., Uria-Nickelsen, M., Thomas, S. R., Trust, T. J. \& Hancock, R. E. (2000). Helicobacter pylori uptake and efflux: basis for intrinsic susceptibility to antibiotics in vitro. Antimicrob Agents Chemother 44, 248-254.

Chopra, I. \& Roberts, M. (2001). Tetracycline antibiotics: mode of action, applications, molecular biology, and epidemiology of bacterial resistance. Microbiol Mol Biol Rev 65, 232-260.

Dailidiene, D., Bertoli, M. T., Miciuleviciene, J., Mukhopadhyay, A. K., Dailide, G., Pascasio, M. A., Kupcinkas, L. \& Berg, D. E. (2002). Emergence of tetracycline resistance in Helicobacter pylori: multiple mutational changes in $16 \mathrm{~S}$ ribosomal DNA and other genetic loci. Antimicrob Agents Chemother 46, 3940-3946.

Falsafi, T., Valizadeh, N., Najafi, M., Ehsani, A., Khani, A., Landarani, Z. \& Falahi, Z. (2007). Culture of Helicobacter pylori from stool samples in children. Can J Microbiol 53, 411-416.

Falsafi, T., Favaedi, R., Mahjoub, F. \& Najafi, M. (2009). Application of stool-PCR test for diagnosis of Helicobacter pylori infection in children. World J Gastroenterol 15, 484-488.

Glocker, E., Berning, M., Gerrits, M. M., Kusters, J. G. \& Kist, M. (2005). Real-time PCR screening for 16S rRNA mutations associated with resistance to tetracycline in Helicobacter pylori. Antimicrob Agents Chemother 49, 3166-3170.

Graham, D. Y. \& Qureshi, W. A. (2000). Antibiotic-resistant H. pylori infection and its treatment. Curr Pharm Des 6, 1537-1544.

Kim, J. J., Reddy, R., Lee, M., Kim, J. G., El-Zaatari, F. A., Osato, M. S. \& Graham, D. Y. (2001). Analysis of metronidazole, clarithromycin and tetracycline resistance of Helicobacter pylori isolates from Korea. J Antimicrob Chemother 47, 459-461.

King, A. (2001). Recommendations for susceptibility tests on fastidious organisms and those requiring special handling. J Antimicrob Chemother 48 (Suppl. S1), 77-80.

Kutschke, A. \& de Jonge, B. L. M. (2005). Compound efflux in Helicobacter pylori. Antimicrob Agents Chemother 49, 3009-3010.

Kwon, D. H., Kim, J. J., Lee, M., Yamaoka, Y., Kato, M., Osato, M. S., El-Zaatari, F. A. K. \& Graham, D. Y. (2000). Isolation and characterization of tetracycline-resistant clinical isolates of Helicobacter pylori. Antimicrob Agents Chemother 44, 3203-3205.

Lawson, A. J., Elviss, N. C. \& Owen, R. J. (2005). Real-time PCR detection and frequency of $16 \mathrm{~S}$ rDNA mutations associated with 
resistance and reduced susceptibility to tetracycline in Helicobacter pylori from England and Wales. J Antimicrob Chemother 56, 282-286.

Li, Y. \& Dannelly, H. K. (2006). Inactivation of the putative tetracycline resistance gene HP1165 in Helicobacter pylori led to loss of inducible tetracycline resistance. Arch Microbiol 185, 255-262.

Lin, J., Michel, L. O. \& Zhang, O. (2002). CmeABC functions as a multidrug efflux system in Campylobacter jejuni. Antimicrob Agents Chemother 46, 2124-2131.

Loffeld, R. J. \& Fijen, C. A. (2003). Antibiotic resistance of Helicobacter pylori: a cross-sectional study in consecutive patients, and relation to ethnicity. Clin Microbiol Infect 9, 600-604.

McNulty, C., Owen, R., Tompkins, D., Hawtin, P., McColl, K., Price, A., Smith, G. \& Teare, L. (2002). Helicobacter pylori susceptibility testing by disk diffusion. J Antimicrob Chemother 49, 601-609.

Mendonca, S., Ecclissato, C., Sartori, M. S., Goday, A. P., Guerrzont, R. A., Degger, M. \& Predrazzoli, J., Jr (2000). Prevalence of Helicobacter pylori resistance to metronidazole, clarithromycin, amoxicillin, tetracycline, and furazolidone in Brazil. Helicobacter 5, 79-83.

Nikaido, H. (1998). Antibiotic resistance caused by Gram-negative multidrug efflux pumps. Clin Infect Dis 27, S32-S41.
Nonaka, L., Connell, S. R. \& Taylor, D. E. (2005). 16S rRNA mutations that confer tetracycline resistance in Helicobacter pylori decrease drug binding in Escherichia coli ribosomes. J Bacteriol 187, 3708-3712.

Osato, M. S. R., Reddy, S. G., Penland, R. I., Malaty, H. M. \& Graham, D. Y. (2001). Pattern of primary resistance of Helicobacter pylori to metronidazole or clarithromucin in the United States. Arch Intern Med 161, 1217-1220.

Trieber, C. A. \& Taylor, D. E. (2002). Mutations in the 16SrRNA genes of Helicobacter pylori mediate resistance to tetracycline. J Bacteriol 184, 2131-2140.

van Amsterdam, K., Bart, A. \& Ende, A. (2005). A Helicobacter pylori TolC efflux pump confers resistance to metronidazole. Antimicrob Agents Chemother 49, 1477-1482.

Wu, H., Shi, X. D., Wang, H. T. \& Liu, J. X. (2000). Resistance of Helicobacter pylori to metronidazole, tetracycline and amoxycillin. J Antimicrob Chemother 46, 121-123.

Wu, J. Y., Kim, J. J., Reddy, K. R., Wang, W. M. \& Graham, D. Y. (2005). Tetracycline-resistant clinical H. pylori isolates with and without mutations in 16rRNA-encoding genes. Antimicrob Agents Chemother 49, 578-583. 